\title{
Clinical Study \\ Cognitive Stimulation for Apathy in Probable Early-Stage Alzheimer's
}

\author{
Linda L. Buettner, ${ }^{1}$ Suzanne Fitzsimmons, ${ }^{1}$ Serdar Atav, ${ }^{2}$ and Kaycee Sink ${ }^{3}$ \\ ${ }^{1}$ The University of North Carolina at Greensboro, HHP 420A, Walker Street, Greensboro, NC 27402, USA \\ ${ }^{2}$ Decker School of Nursing, Binghamton University, Binghamton, NY 13906, USA \\ ${ }^{3}$ Sticht Center on Aging, Wake Forest University Baptist Medical Center, Winston-Salem, NC 27157, USA
}

Correspondence should be addressed to Linda L. Buettner, llbuettn@uncg.edu

Received 9 December 2010; Accepted 2 March 2011

Academic Editor: Willem A. Van Gool

Copyright (C) 2011 Linda L. Buettner et al. This is an open access article distributed under the Creative Commons Attribution License, which permits unrestricted use, distribution, and reproduction in any medium, provided the original work is properly cited.

\begin{abstract}
We studied changes in apathy among 77 community-dwelling older persons with mild memory loss in a randomized clinical trial comparing two nonpharmacological interventions over four weeks. The study used a pre-post design with randomization by site to avoid contamination and diffusion of effect. Interventions were offered twice weekly after baseline evaluations were completed. The treatment group received classroom style mentally stimulating activities (MSAs) while the control group received a structured early-stage social support (SS) group. The results showed that the MSA group had significantly lower levels of apathy $(P<.001)$ and significantly lower symptoms of depression $(P<.001)$. While both groups improved on quality of life, the MSA group was significantly better $(P=.02)$ than the SS group. Executive function was not significantly different for the two groups at four weeks, but general cognition improved for the MSA group and declined slightly for the SS group which produced a significant posttest difference $(P<.001)$. Recruitment and retention of SS group members was difficult in this project, especially in senior center locations, while this was not the case for the MSA group. The examination of the data at this four-week time point shows promising results that the MSA intervention may provide a much needed method of reducing apathy and depressive symptoms, while motivating participation and increasing quality of life.
\end{abstract}

\section{Introduction}

Neuropsychiatric symptoms of dementia are common and associated with poor outcomes for patients and caregivers [1]. Apathy is the most common neuropsychiatric symptom in mild to moderate stages of Alzheimer's disease (AD) and increases in severity as the dementia progresses. Apathy is complex to treat in $\mathrm{AD}$ since it is often mixed with other challenging behaviors and confused with depression [2]. A review of scientific evidence shows that pharmacological therapies are not particularly effective for management of these mixed symptoms and further complicated by dangerous side effects [3]. With an estimated 5.5 million Americans with dementia, safe, effective, and easy to deliver therapies are desperately needed for this problem [1,3-5].

Apathy is defined as the loss of motivation not attributable to cognitive impairment, emotional distress, or reduced level of consciousness [5]. Apathy occurs in approximately $70 \%$ of Alzheimer's disease (AD) patients within five years of diagnosis [6]. AD patients with apathy require more management and support, given their reliance on others to schedule their activities and initiate tasks even when they are still physically capable of performing them. Studies examining the relationship of apathy to neuropsychological measures showed that apathy was consistently associated with more severe functional impairments, more severe cognitive deficits, higher levels of burden and distress in caregivers, along with increased resource utilization $[7,8]$.

Apathy in early-stage AD is often overlooked by healthcare providers, yet if left untreated, leads to more rapid than expected functional decline [9]. The etiology of apathy is complex and thought to be related to underlying pathology, atrophy of the frontal lobes, and their connections to the temporal lobes [10]. Damage to these areas of the brain 


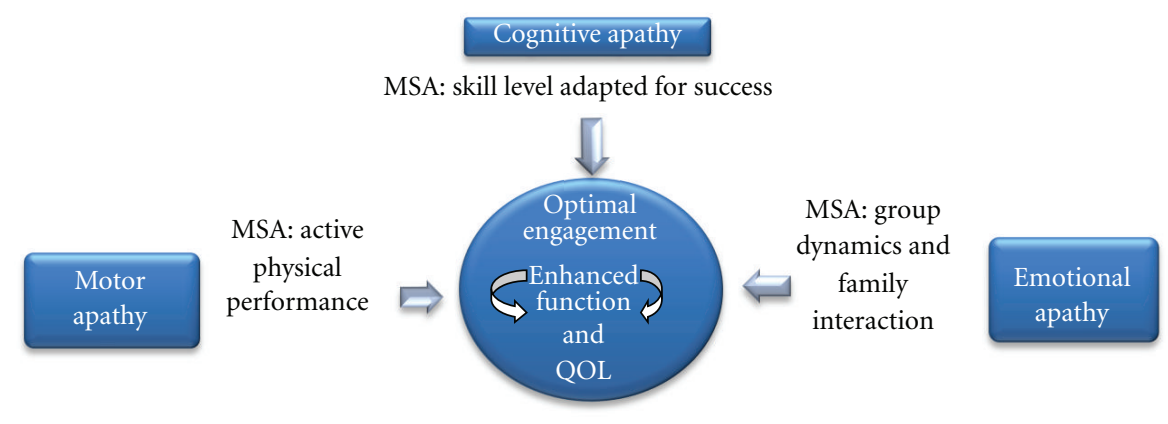

FIgURE 1: Theoretical model for apathy and the MSA intervention.

reduces the ability to initiate, sequence, and complete tasks. Such losses can lead to unmet needs for cognitive, social, emotional, and physical stimulation.

Older individuals with early-stage memory problems have unmet needs with respect to stimulation, socialization, needs for adaptation of tasks for successful performance, and yet have a desire for novel mentally stimulating experiences to maintain abilities $[2,11-16]$. Research shows that patients with Mild Cognitive Impairment (MCI) and early-stage AD continue to respond well to stimulation, often performing at the same levels of brain activity as age-matched controls $[17,18]$. While the current "state-of-the-art" intervention for early-stage dementia is an early stage support group, those with apathy may not attend or may not benefit in the same way as more motivated participants.

Expanding on Marin's Model of Apathy [19] (Figure 1), we hypothesize that Mentally Stimulating Activities (MSAs) that match current skill levels should be more effective in engaging participants with apathy and early-stage memory loss because they meet individual needs for stimulation while leading to more focused and successful therapeutic experiences. Further, when engaged in MSAs with others, in classroom-style groups, positive emotions should increase and pleasant experiences with others occur. Finally, the physical performance that takes place in each MSA session reduces motor apathy and provides game-like opportunities for active engagement again. These three subcomponents work together to reduce motor, cognitive, and emotional apathy, allow for failure-free enhanced engagement with others, and theoretically could help participants maintain function and improve QOL. This randomized controlled trial (RCT) offered participants either a course in classroom style MSAs or a structured early-stage social support (SS) group for symptoms of early-stage memory loss. This paper will present the impact of a 2-arm RCT cognitive stimulation intervention on apathy, depression, executive function, and cognition in a subset of participants after 4 weeks of intervention. The fully powered complete study will have 120 participants with repeated measures at baseline, four weeks, eight weeks, and 12 weeks.

\section{Methods}

2.1. Study Design and Participants. The first 77 participants randomized in the trial with pretest and 4-week posttest scores are included in this paper. Randomization by site ( $n=8$ sites) rather than by participant was used to control for possible cross-contamination of treatment or diffusion of effect. The participants to date were recruited from Continuing Care Retirement Communities and local senior centers in central North Carolina and met the following inclusion criteria: English speaking; 60 years of age or older; diagnosis of dementia or symptoms that meet DSM-IV [20] criteria for probable early-stage AD or Peterson criteria for MCI [21]; a Mini-Mental State Exam (MMSE) [22] score of 18 or greater but less than 28 ; stable on any psychoactive drug from prebaseline through final observation to control for the effect these drugs have on neuropsychiatric behavior; and presence of apathy as reported by family, significant other, or medical provider. Exclusion criteria included delirium or a progressive, unstable medical, metabolic, or neurological illness that might interfere with behavior; recent fracture (within 4 months); history of Parkinson's disease, Huntington's disease, seizure disorder, major stroke, alcoholism, drug abuse, recent head trauma with loss of consciousness, or psychiatric illness preceding the onset of memory loss; severe vision or hearing impairment; and receiving a new psychoactive medication within the past 30 days. Participants were screened for inclusion by a Geriatric Nurse Practitioner, and for cases without cognitive diagnoses the study PI or medical director was consulted before the participant was included. The IRB of the University of North Carolina at Greensboro provided human subjects approval and data safety and monitoring for this study.

2.2. Mentally Stimulating Activity Program. The MSA program was developed by Fitzsimmons $[23,24]$ and has been used in community-based, hospital, and long-term care settings by the first and second authors in clinical practice. Specific cognitive tasks target attention, orientation, concentration, short-term memory, and organized thinking in a classroom style setting. In general, activities were designed to be stimulating, challenging, novel, and fun but not frustrating. Each task takes from 2-10 minutes and is interactive and goal driven. The one hour, twice weekly sessions included a variety of physical and cognitive activities that rely on various functions of the brain: visual spatial abilities, verbal and nonverbal communication skills, abstract concepts, eye-hand coordination, fine motor control, body orientation, crossing the midline, recognition memory, short-and long-term 
TABle 1: Participant demographics (values are presented as $n(\%)$ or mean (standard deviation)).

\begin{tabular}{|c|c|c|c|c|}
\hline & Total & Control (social support) & Treatment (mentally stimulating activities) & $P$ value \\
\hline Participants $(n)$ & 77 & $29 *$ & 48 & \\
\hline Gender & & & & .243 \\
\hline Male & $15(19.5 \%)$ & $4(13.8 \%)$ & $11(22.9 \%)$ & \\
\hline Female & $62(80.5 \%)$ & $25(86.2 \%)$ & $36(77.1 \%)$ & \\
\hline Race & & & & .071 \\
\hline Caucasian & $56(72.7 \%)$ & $17(58.6 \%)$ & $39(81.3 \%)$ & \\
\hline African-American & $20(25.9 \%)$ & $11(37.9 \%)$ & $9(18.7 \%)$ & \\
\hline Hispanic & $1(1.3 \%)$ & $1(.03 \%)$ & $0(0 \%)$ & \\
\hline Depression $D X$ & & & & .594 \\
\hline Yes & 20 & $7(24.1 \%)$ & $13(27.1 \%)$ & \\
\hline No & 57 & $22(75.9 \%)$ & $35(72.9 \%)$ & \\
\hline Mean Age & & $82.2(6.5)$ & $81.0(8.7)$ & .07 \\
\hline Years of Education & & $13.6(3.8)$ & $14.6(3.6)$ & .499 \\
\hline \multicolumn{5}{|l|}{ Baselines } \\
\hline MMSE & & $25.4(2.8)$ & $25.2(3.3)$ & .269 \\
\hline AES (Apathy) & & $30.4(7.9)$ & $32.8(8.7)$ & .331 \\
\hline Cornell-Brown & & $18.6(11.3)$ & $14.3(10.8)$ & .758 \\
\hline PHQ-9 & & $4.7(4.8)$ & $5.9(5.7)$ & .188 \\
\hline Trail Making Time & & $192.80(89.7)$ & $178.45(90.0)$ & .635 \\
\hline
\end{tabular}

* 48 participants were enrolled but 19 dropped after baseline recruitment and evaluation due to health problems and lack of interest in the intervention (39\%). These participants were not included in the pre-post analysis.

memory, executive functioning, and sensory identification. The facilitated tasks were performed by participants alone, in pairs, or as a group. Participants were encouraged to find alternate methods to complete the tasks and were encouraged to help each other.

2.3. Structured Early-Stage Social Support Program. The SS program was developed as a structured psychoeducational group with information about brain health, group discussion, and emotional support provided during each session. The trained facilitator provided the structure and led the discussion. Group members were encouraged to share frustrations, concerns, and thoughts on dealing with the emotional, social, and everyday problems that accompany memory loss. Sessions were provided twice weekly for one hour to provide an equivalent amount of social contact.

\section{Data Collection}

The following measures of psychological and cognitive functioning were obtained at baseline and after four weeks of the intervention by trained research assistants who were blinded to the participant's group assignment: apathy was measured using the Apathy Evaluation Scale (AES) [19]. The AES is an 18-item scale measuring things such as interests, starting and completing things, and initiating activities. Internal consistency is good, with an alpha ranging of $0.86-0.94$. Depressive symptoms were measured with the Patient Health Questionnaire (PHQ-9) [25]. The PHQ-9 is a 9-item scale with scores $\geq 10$ having a sensitivity of $88 \%$ and a specificity of $88 \%$ for major depression. The CornellBrown QOL [26] was used to measure quality of life.
Interrater reliability (intraclass $r=0.90$ ) and internal consistency reliability (Cronbach alpha $=0.81$ ) are very good. Global cognition was measured with the Mini-Mental State Examination (MMSE) which consists of 11 simple questions or tasks. Test-retest reliability is excellent $(r>$ 0.89 ). Attention and executive functions were measured with the Trail Making test part B [27] (Table 3). Table 2 displays evaluation tools used in the study.

3.1. Statistical Methods. Chi-Square tests and $t$-tests were used to describe the participants by treatment assignment at baseline. To determine the impact of the intervention on outcomes at four weeks, Analysis of Covariance (ANCOVA) was used. Between group differences are presented and adjusted for baseline scores. Statistical analyses were performed with PASW Statistics 18 software. Statistical significance for the study was established at $P<.05$.

\section{Results}

Demographics of the first 77 participants are listed in Table 1 . The control group (structured social support) had an attrition of eighteen participants (37\%) after baseline screening and before the posttest evaluations took place. The treatment group (mentally stimulating activities) had only one dropout thus the groups reported in this paper are uneven with 29 in control and 48 in treatment due to high SS attrition after intervention assignment. Examining the participants that remained in the study for four weeks, we found no significant difference between groups in baseline age, education, apathy, depression, or MMSE scores. The treatment group had slightly more depressive symptoms on the PHQ-9, slightly 
TABLE 2: Variables and measurement.

\begin{tabular}{|c|c|c|}
\hline $\begin{array}{l}\text { Cognition and Eligibility } \\
\text { for study }\end{array}$ & $\begin{array}{l}\text { Mini-Mental State examination } \\
\text { (MMSE) [22] }\end{array}$ & $\begin{array}{l}\text { The MMSE consists of } 11 \text { simple questions or tasks. } \\
\text { Test-retest reliability }(r>0.89) \text {. }\end{array}$ \\
\hline Neuropsychiatric behaviors & $\begin{array}{l}\text { Clinician administered Apathy } \\
\text { Evaluation Scale (AES) [19] }\end{array}$ & $\begin{array}{l}\text { The AES is an } 18 \text {-item scale. Internal consistency has an } \\
\text { alpha range of } 0.86-0.94 \text {. Validity: differences found } \\
P<.05\end{array}$ \\
\hline Quality of life & Cornell-Brown QOL [24] & $\begin{array}{l}\text { Interrater reliability (intraclass } r=0.90) \text { and internal } \\
\text { consistency reliability }(\text { Cronbach alpha }=0.81) .\end{array}$ \\
\hline Depression & $\begin{array}{l}\text { The Patient Health Questionnaire [25] } \\
\text { (PHQ-9) }\end{array}$ & $\begin{array}{l}\text { Nine-item scale with PHQ- } 9 \text { score } \geq 10 \text { had a sensitivity of } \\
88 \% \text { and a specificity of } 88 \% \text { for major depression. }\end{array}$ \\
\hline Executive function & Trail Making B [26] & $\begin{array}{l}\text { Validity is high, especially when measuring attention and } \\
\text { executive function }\end{array}$ \\
\hline
\end{tabular}

TABLE 3: Analyses of covariance summary table for the effect of treatment.

\begin{tabular}{|c|c|c|c|c|c|c|}
\hline & & Adjusted means at baseline & Adjusted means at 4 weeks & $n$ & $F$ & Significance ( $P$ value) \\
\hline \multirow{2}{*}{ Apathy } & SS & 30.38 & 35.52 & 29 & \multirow{2}{*}{31.496} & \multirow{2}{*}{$<.001$} \\
\hline & MSA & 32.08 & 28.19 & 48 & & \\
\hline \multirow{2}{*}{ Cornell Brown QOL } & SS & 18.62 & 20.52 & 29 & \multirow{2}{*}{4.978} & \multirow{2}{*}{.029} \\
\hline & MSA & 14.31 & 22.92 & 48 & & \\
\hline \multirow{2}{*}{ MMSE } & SS & 25.41 & 24.79 & 29 & \multirow{2}{*}{22.429} & \multirow{2}{*}{$<.001$} \\
\hline & MSA & 25.17 & 26.10 & 48 & & \\
\hline \multirow{2}{*}{ PHQ-9 } & SS & 4.66 & 6.48 & 29 & \multirow{2}{*}{13.319} & \multirow{2}{*}{$<.001$} \\
\hline & MSA & 5.94 & 4.55 & 48 & & \\
\hline \multirow{2}{*}{ Trail making } & SS & 192.20 & 178.89 & 29 & \multirow{2}{*}{.022} & \multirow{2}{*}{.258 (n.s) } \\
\hline & MSA & 184.73 & 161.42 & 48 & & \\
\hline
\end{tabular}

more apathy, and slightly poorer quality of life reported on the Cornell-Brown Scale prior to intervention.

After four weeks of intervention, the participants in the MSA group had significantly lower apathy scores than those in the SS group. Specifically, the MSA group dropped 3.9 points on the Apathy Evaluation Scale-Clinical Version and the SS group increased 5.1 points $(P=.001)$. Quality of life as measured by the Cornell Brown Scale improved for both groups but the MSA group was significantly better $(P<.02)$. The MSA group improved 8.62 points, and the SS group improved 1.9 points. Participants in the MSA group improved by .93 on the MMSE $(P=.001)$ and the SS group declined by .62 indicating a significant improvement in cognition occurred for the MSA group after removing the influence of pretest scores. While 57 participants did not indicate depression diagnosis at baseline, a significant change occurred in both groups on the PHQ-9 after four weeks. Participants in the MSA group had a 1.39 point drop, and the SS group had a 1.82 point increase in depression scores $(P<.001)$ after removing the influence of pretest scores. Neither group was significantly different on the Trail Making test.

\section{Discussion}

In this four-week study of 77 participants with earlystage memory loss and apathy, those taking part in the MSA sessions improved significantly in the areas of apathy, depressive symptoms, and quality of life in comparison to those in the structured social support sessions. A small improvement in mean MMSE score also occurred for the MSA group but the primary target for this study was apathy. We did not find any difference in executive function at week four, but perhaps the intervention will take longer to affect a change in executive function. These improvements are consistent with the findings of earlier studies [28, 29]. Our focus on reducing apathy may provide a novel therapeutic route to improving mood and quality of life, and maintaining cognitive function over time. The early-stage support group experienced a $37 \%$ attrition rate, and for those that remained at the four week posttest, there was a significant increase in apathy symptoms. These apathy symptoms could explain poorer performance on the evaluation scales.

Most cognitive stimulation or cognitive training trials have targeted healthy older adults and have found improvements on specific tasks [30]. This trial took a different approach with the focus on breaking the pattern of apathy during early stages of memory loss, which other studies have shown to lead to premature functional decline. For people with Alzheimer's disease, cognitive stimulation therapy that provides activities involving cognitive processing, within a social context, with an emphasis on enjoyment holds great promise.

There were a number of limitations. It should be noted that this study was limited due to the rigorous inclusion criteria which were necessary to ensure a homogeneous group of participants. This meant many participants with 
related neurological conditions and complex health problems were excluded. Another limitation was the study sites which were selected by socioeconomic status (SES) (four high SES and four middle to low SES), which allowed diverse senior centers with 3-7 candidates to participate. These small groups enhanced project diversity enrollment but provided less than ideal program sizes which may have influenced outcomes. Recruitment and retention in lower SES sites was a problem. Although groups of 10 were recruited in senior centers, participants did not attend routinely, preferring to take part in other activities or simply not attending after group assignment. Several efforts were made to encourage participation with phone followups, discussions with family or staff members at the sites, but the attrition rate for the control condition was much higher than expected.

In clinical practice, outside of a research trial, participants and groups would be best selected through clinical judgment, considering complexities like sensory impairment, communication deficits, interest, and mix of participants instead of SES and diversity recruitment. In the randomization procedure, ideally the generation of the allocation sequence, enrollment into the trial, and allocation to group should be separate and performed by different, independent staff. The PI and Project Director were directly involved with this process with the statistician due to an initial budget cut from the trial funder.

Many more people are being diagnosed earlier in the course of memory loss because of improving sensitivity and specificity of diagnostic techniques and increased awareness of Alzheimer's disease and related disorders. Medical, nursing, allied health professionals, the Alzheimer's Association, and other organizations are responding by offering a range of support groups to ease the social strains of people with earlystage dementia. While these support services are needed, it appears that the MSA cognitive stimulation intervention program may be another important option for individuals with early-stage memory changes, especially if they also have apathy symptoms. Offering this type of cognitive stimulation could influence the course of the disease overtime as our early findings show individuals with early-stage memory loss and apathy perform well, improve, and continue to eagerly attend twice per week MSA program sessions.

\section{Conclusions}

Over $70 \%$ of people with early-stage Alzheimer's disease experience apathy. This symptom is rarely indentified or treated, which inhibits the older adult's ability to remain active with intellectual, interpersonal, and physical activities. In light of the high prevalence of apathy in dementia and its impact on quality of life among older people, the use of MSA cognitive stimulation groups as a means of reducing apathy and depressive symptoms, as well as increasing quality of life is very appealing. Therapists, nurses, and other aging services professionals in community settings can incorporate classroom style cognitive stimulation sessions for clients with dementia and apathy who are underperforming or refusing to participate in other types of therapeutic programs.

\section{Acknowledgments}

The authors wish to thank all who kindly participated in the study in NC, UNCG IRB for compliance review and data safety monitoring, and Alzheimer's Association for funding this study (Grant IIRG-09-131429).

\section{References}

[1] L. E. Hebert, P. A. Scherr, J. L. Bienias, D. A. Bennett, and D. A. Evans, "Alzheimer disease in the US population: prevalence estimates using the 2000 census," Archives of Neurology, vol. 60, no. 8, pp. 1119-1122, 2003.

[2] L. Buettner, S. Fitzsimmons, and S. Atav, "Predicting outcomes of therapeutic recreation interventions for older adults with dementia and behavioral symptoms," Therapeutic Recreation Journal, vol. 40, no. 1, pp. 33-47, 2006.

[3] K. M. Sink, K. F. Holden, and K. Yaffe, "Pharmacological treatment of neuropsychiatric symptoms of dementia: a review of the evidence," Journal of the American Medical Association, vol. 293, no. 5, pp. 596-608, 2005.

[4] L. Buettner and S. Fitzsimmons, "Mixed behaviors in dementia: the need for a paradigm shift," Journal of Gerontological Nursing, vol. 32, no. 7, pp. 15-22, 2006.

[5] R. S. Marin, "Apathy: a neuropsychiatric syndrome," Journal of Neuropsychiatry and Clinical Neurosciences, vol. 3, no. 3, pp. 243-254, 1991.

[6] P. A. Boyle and P. F. Malloy, "Treating apathy in Alzheimer's disease," Dementia and Geriatric Cognitive Disorders, vol. 17, no. 1-2, pp. 91-99, 2004.

[7] S. E. Starkstein, L. Ingram, M. L. Garau, and R. Mizrahi, "On the overlap between apathy and depression in dementia," Journal of Neurology, Neurosurgery and Psychiatry, vol. 76, no. 8, pp. 1070-1074, 2005.

[8] A. M. Landes, S. D. Sperry, M. E. Strauss, and D. S. Geldmacher, "Apathy in Alzheimer's disease," Journal of the American Geriatrics Society, vol. 49, no. 12, pp. 1700-1707, 2001.

[9] S. E. Starkstein, R. Jorge, R. Mizrahi, and R. G. Robinson, "A prospective longitudinal study of apathy in Alzheimer's disease," Journal of Neurology, Neurosurgery and Psychiatry, vol. 77, no. 1, pp. 8-11, 2006.

[10] R. Levy and B. Dubois, "Apathy and the functional anatomy of the prefrontal cortex-basal ganglia circuits," Cerebral Cortex, vol. 16, no. 7, pp. 916-928, 2006.

[11] S. C. Burgener, L. L. Buettner, K. Coen Buckwalter et al., "Evidence supporting nutritional interventions for persons in early stage Alzheimer's disease (AD)," Journal of Nutrition, Health and Aging, vol. 12, no. 1, pp. 18-21, 2008.

[12] L. Buettner, "Community-based RT. Peace of mind: a pilot community-based program for older adults with memory loss," Journal of Recreation Therapy, pp. 42-48, 2006.

[13] S. Fitzsimmons and L. L. Buettner, "Therapeutic recreation interventions for need-driven dementia-compromised behaviors in community-dwelling elders," American Journal of Alzheimer's Disease and other Dementias, vol. 17, no. 6, pp. 367-381, 2002.

[14] R. Verkaik, J. C. M. van Weert, and A. L. Francke, "The effects of psychosocial methods on depressed, aggressive and apathetic behaviors of people with dementia: a systematic review," International Journal of Geriatric Psychiatry, vol. 20, no. 4, pp. 301-314, 2005. 
[15] L. Buettner and S. Fitzsimmons, "Promoting health in Alzheimer's disease: evaluation of a 12-week course for people with early stage dementia," Journal of Gerontological Nursing. In press.

[16] A. M. Kolanowski, L. Buettner, D. M. Fick, S. Fitzsimmons, and M. Cornacchione, "Instituting cognitive rehabilitation in post-acute care," Annals of Long-Term Care, vol. 16, no. 2, pp. 40-46, 2008.

[17] R. L. Gould, B. Arroyo, R. G. Brown, A. M. Owen, E. T. Bullmore, and R. J. Howard, "Brain mechanisms of successful compensation during learning in Alzheimer disease," Neurology, vol. 67, no. 6, pp. 1011-1017, 2006.

[18] S. I. Rapoport, "Functional brain imaging in the resting state and during activation in Alzheimer's disease. Implications for disease mechanisms involving oxidative phosphorylation," Annals of the New York Academy of Sciences, vol. 893, pp. 138153, 1999.

[19] R. S. Marin, "Apathy: a neuropsychiatric syndrome," Journal of Neuropsychiatry and Clinical Neurosciences, vol. 3, no. 3, pp. 243-254, 1991.

[20] American Psychiatric Association, Diagnostic and Statistical Manual of Mental Disorders, American Psychiatric Association, Washington, DC, USA, 4th edition, 1994.

[21] B. Winblad, K. Palmer, M. Kivipelto et al., "Mild cognitive impairment-beyond controversies, towards a consensus: report of the International Working Group on Mild Cognitive Impairment," Journal of Internal Medicine, vol. 256, no. 3, pp. 240-246, 2004.

[22] M. F. Folstein, S. E. Folstein, and P. R. McHugh, “'Mini mental state'. A practical method for grading the cognitive state of patients for the clinician," Journal of Psychiatric Research, vol. 12, no. 3, pp. 189-198, 1975.

[23] S. Fitzsimmons, Brain Fitness, Venture, State College, Pa, USA, 2008.

[24] S. Fitzsimmons, "Brain fitness for older adults with dementia," Activities Directors' Quarterly, vol. 8, no. 2, pp. 36-40, 2007.

[25] R. E. Ready and B. R. Ott, "Quality of life measures for dementia," Health and Quality of Life Outcomes, vol. 1, article no. 11, 2003.

[26] K. Kroenke, R. L. Spitzer, and J. B. W. Williams, "The PHQ9: validity of a brief depression severity measure," Journal of General Internal Medicine, vol. 16, no. 9, pp. 606-613, 2001.

[27] J. D. Corrigan and N. S. Hinkeldey, "Relationships between Parts A and B of the Trail Making Test," Journal of Clinical Psychology, vol. 43, no. 4, pp. 402-409, 1987.

[28] V. Breuil, J. De Rotrou, F. Forette et al., "Cognitive stimulation of patients with dementia: preliminary results," International Journal of Geriatric Psychiatry, vol. 9, no. 3, pp. 211-217, 1994.

[29] R. T. Woods, "Psychological 'therapies' in dementia," in Handbook of the Clinical Psychology of Ageing, R. T. Woods, Ed., pp. 575-600, John Wiley \& Sons, Chichester, UK, 1996.

[30] S. L. Willis, S. L. Tennstedt, M. Marsiske et al., "Long-term effects of cognitive training on everyday functional outcomes in older adults," Journal of the American Medical Association, vol. 296, no. 23, pp. 2805-2814, 2006. 


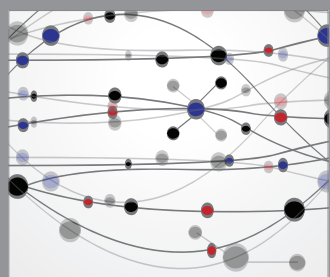

The Scientific World Journal
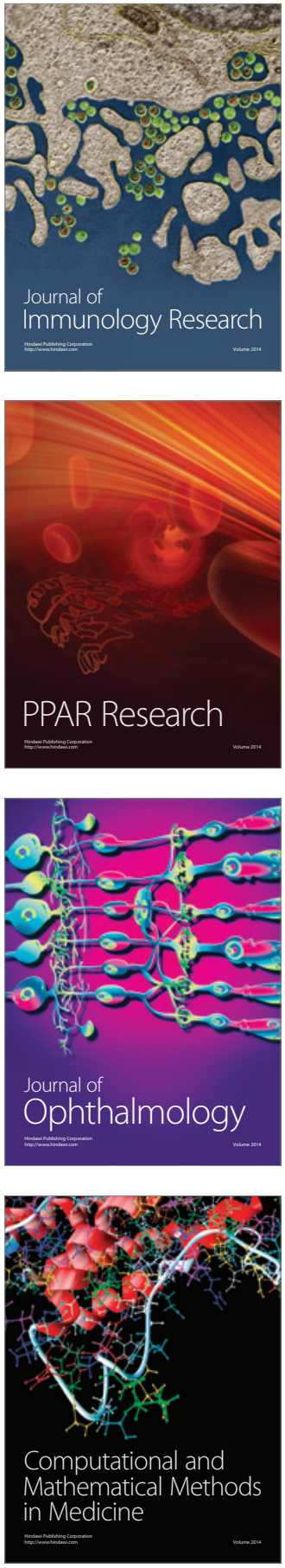

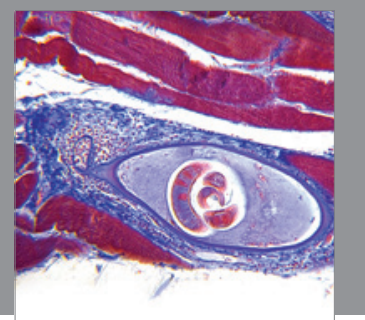

Gastroenterology

Research and Practice
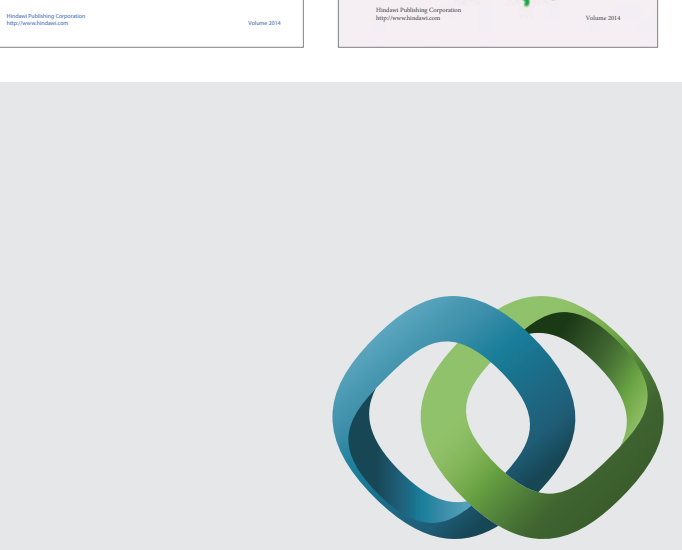

\section{Hindawi}

Submit your manuscripts at

http://www.hindawi.com
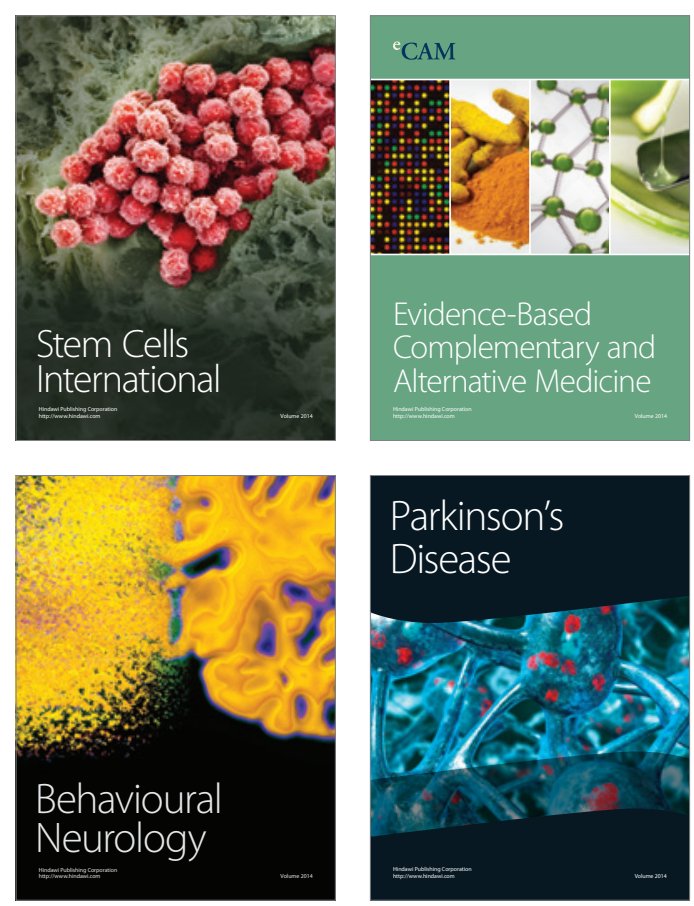

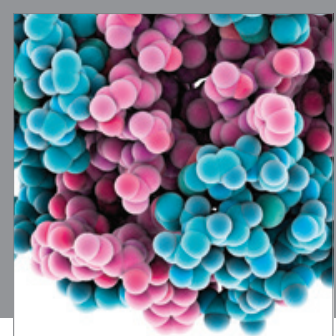

Journal of
Diabetes Research

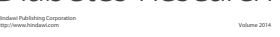

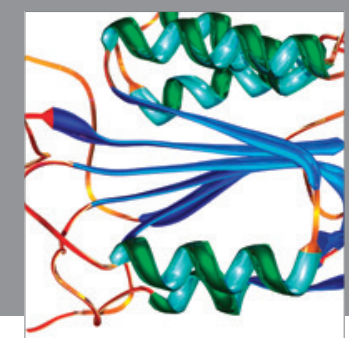

Disease Markers
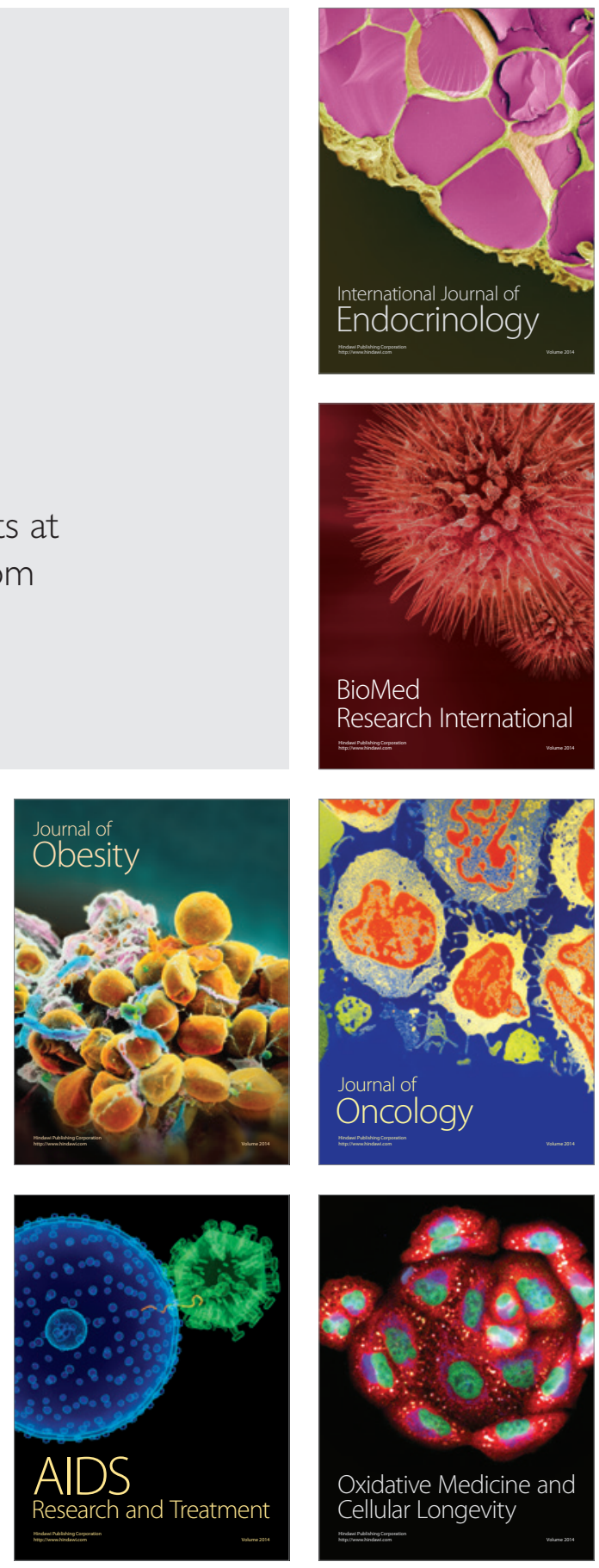